\title{
Review Article \\ Performance and Trends in Millimetre-Wave CMOS Oscillators for Emerging Wireless Applications
}

\author{
Marius Voicu, ${ }^{1,2}$ Domenico Pepe, ${ }^{1}$ and Domenico Zito ${ }^{1,2}$ \\ ${ }^{1}$ Tyndall National Institute, Lee Maltings, Dyke Parade, Cork, Ireland \\ ${ }^{2}$ Department of Electrical and Electronic Engineering, University College Cork, Cork, Ireland \\ Correspondence should be addressed to Domenico Zito; domenico.zito@tyndall.ie
}

Received 14 December 2012; Accepted 19 February 2013

Academic Editor: Ramesh Pokharel

Copyright (c) 2013 Marius Voicu et al. This is an open access article distributed under the Creative Commons Attribution License, which permits unrestricted use, distribution, and reproduction in any medium, provided the original work is properly cited.

\begin{abstract}
This paper reports the latest advances on millimeter-wave CMOS voltage-controlled oscillators (VCOs). Current state-of-the-art implementations are reviewed, and their performances are compared in terms of phase noise and figure of merit. Low power and low phase noise LC-VCO and ring oscillator designs are analyzed and discussed. Design and performance trends over the last decade are provided and discussed. The paper shows how for the higher range of millimeter-waves $(>60 \mathrm{GHz})$ the performances of ring oscillators become comparable with those of LC-VCOs.
\end{abstract}

\section{Introduction}

In the last few years several standards have been, or have been planned to soon be, released, regarding millimetre-waves (mm-waves, i.e., $30-300 \mathrm{GHz}$ ) systems for emerging wireless applications. Some of the most attractive applications are $60 \mathrm{GHz}$ unlicensed wireless data communication [1], 77-GHz automotive radars [2], and $94 \mathrm{GHz}$ passive imaging [3]. Key enabler for high-volume and low-cost mass market implementation of these systems is the significant improvement of device performance in the latest CMOS technology nodes (i.e., $130 \mathrm{~nm}$ and smaller), which offer a great potential for the realization of millimeter-waves wireless transceivers on a single chip.

One of the most important building blocks in a wireless transceiver is the frequency synthesizer. Performance of the voltage controlled oscillator (VCO) dictates the performance of the frequency synthesizer and thus of the whole communication system.

The aim of the present paper is to provide a review of the state-of-the-art (SoA) of millimeter-wave (mm-wave, 30$300 \mathrm{GHz}$ ) VCOs in CMOS technology in order to identify the trends over the last decade and derive some useful observations regarding the past and possible future evolution of design and performance. In particular, the paper reports a comparison of performances among SoA design solutions and highlights the achievements and trends in terms of phase noise $(\mathrm{PN})$ and figure of merit (FOM).

The present paper is organized as follows. Section 2 provides an overview of two of the most widespread VCO topologies, LC-tank, and ring oscillators and recalls briefly their main causes responsible for the phase noise. In Section 3, SoA millimeter-wave CMOS LC-VCO and ring oscillator design solutions are reported, and their performances are discussed and compared. In Section 4, the conclusions are drawn.

\section{CMOS VCOs}

The most widespread CMOS VCO topologies at mm-wave frequencies are LC-tank and ring oscillators. Section 2.1 provides a brief overview of LC-VCOs and their PN contributions. Section 3.1 provides a brief review of ring oscillators and their PN contributions.

2.1. LC-Tank VCOs. LC-VCOs consist of a resonant circuit (LC-tank) and an amplifier that provides adequate gain to compensate the losses of the resonant circuit. The amplifier can be a single transistor in one of the known configurations (common-source, common-gate, or source follower) or the widespread cross-coupled differential pair (see Figure 1(a)). 


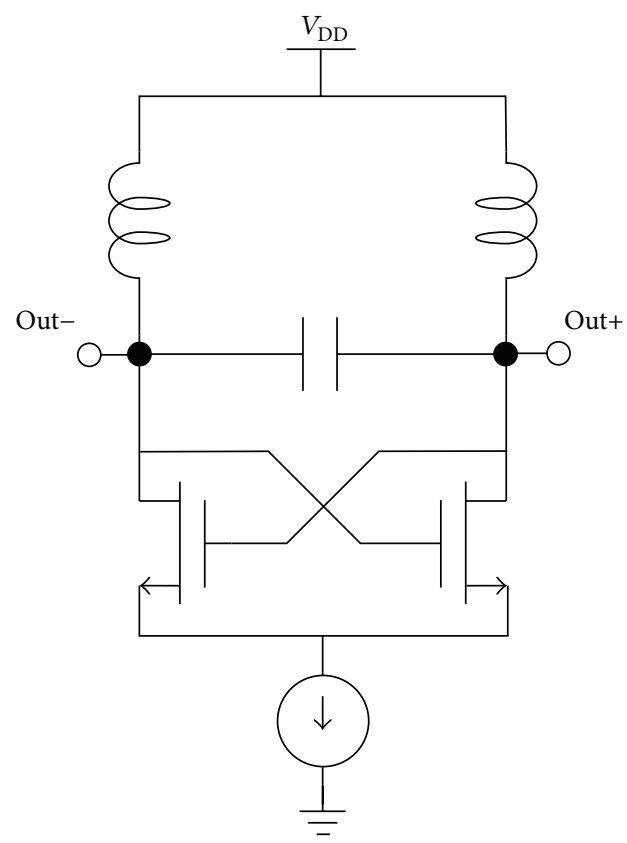

(a)

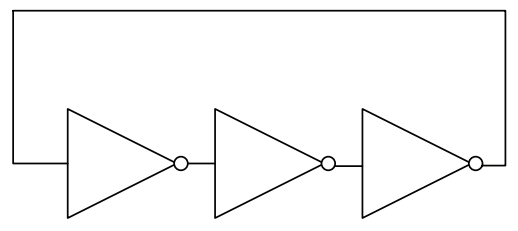

(b)

FIGURE 1: (a) LC-VCO; (b) ring oscillator.

The main causes of PN in LC-VCO are due to the losses in the resonator and the amplifier noise. For instance, in the case of cross-coupled differential pair LC-VCOs, they are (i) resonator thermal noise (due to the loss conductance in the resonator), (ii) tail current noise (the switching action of the differential pair translates noise up and down in frequency, and so the noise enters the resonator), and (ii) differential pair noise (due to the finite switching time of the pair) [4].

2.2. Ring Oscillators. Ring oscillators (ROs) are composed of a cascade of inverting amplifiers, and the output of the last element is fed back to the input of the first (see Figure 1(b)). These inverter stages can be implemented by differential amplifiers, CMOS inverters, or even LC-VCOs.

The main causes of PN in ring oscillators are (i) the thermal noise (due to MOSFET drain-source channel resistance and load resistors) and (ii) flicker noise (in CMOS inverterbased ROs, the pull-up and pull-down currents contain flicker noise which varies slowly over many transitions, while, in differential ROs, the flicker noise in the tail current modulates the propagation delay of the stages) [5].

\section{State-of-the-Art of mm-Wave CMOS VCOs}

In this Section, a review of SoA mm-wave CMOS LC-VCO and $\mathrm{RO}$ design solutions is provided, and their performances are discussed and compared. In Section 3.1, three SoA mmwaves CMOS LC-VCO implementations, operating at 30, 60 , and $140 \mathrm{GHz}$, respectively, are reported. In Section 3.2, two SoA mm-wave RO designs (the first implemented at 50 and $60 \mathrm{GHz}$, the second at 104 and $121 \mathrm{GHz}$ ) are reported. In Section 3.3, performance trends over the last decade of

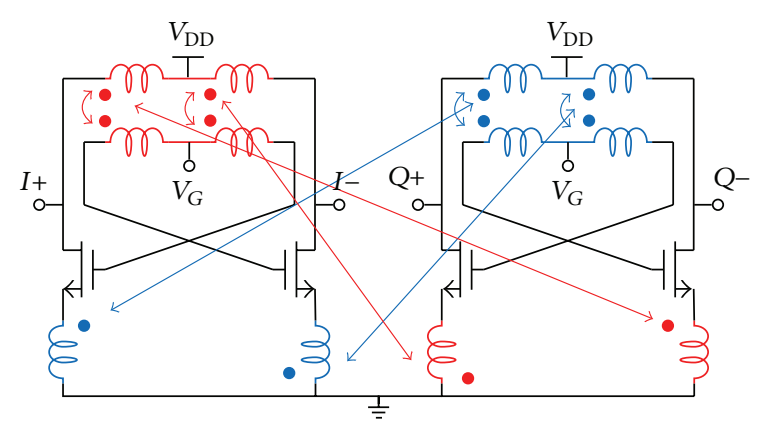

FIgURE 2: Schematic of the VCO presented in [6]. The two trifilar transformers of $I$ and Q VCOs are highlighted in red and blue. Spirals couplings are represented by the thin arrowed lines. $I$ and $Q$ are VCO outputs.

mm-wave CMOS LC-VCOs and ROs are provided and discussed. The performances of the SoA VCOs are summarized in Tables 1 and 2, and their figure of merit ((FOM) see (1)) are evaluated:

$$
\mathrm{FOM}=P N-20 \log \left(\frac{f_{0}}{\Delta f}\right)+10 \log \left(\frac{P_{\mathrm{DC}}}{1 \mathrm{~mW}}\right),
$$

where $f_{0}$ is the oscillation frequency, $\Delta f$ is the offset at which the $P N$ is evaluated, and $P_{C}$ is the power consumption.

3.1. SoA mm-Wave CMOS LC-VCOs. In [6] a $30 \mathrm{GHz}$ quadrature VCO (QVCO) implemented in $0.13 \mu \mathrm{m}$ CMOS technology is presented. The circuit schematic is shown in Figure 2. It exploits the use of a trifilar $(1: 1: 1)$ transformer with a high quality factor (i.e., with respect to spiral inductors) 
TABLE 1: SoA mm-wave CMOS LC-VCOs: summary of performance.

\begin{tabular}{lcccccc}
\hline Reference & Tech. $(\mathrm{nm})$ & $f_{0}(\mathrm{GHz})$ & $V_{\mathrm{DD}}(\mathrm{V})$ & $P_{\mathrm{C}}(\mathrm{mW})$ & Phase noise $(\mathrm{dBc} / \mathrm{Hz})$ & $\mathrm{FOM}(\mathrm{dBc} / \mathrm{Hz})$ \\
\hline$[6]$ & 130 & 30.3 & 0.6 & 7.8 & $-114 @ 1 \mathrm{MHz}$ & -196 \\
{$[7]$} & 90 & 57.6 & 0.6 & 7.2 & $-102 @ 1 \mathrm{MHz}$ & -188 \\
{$[9]$} & 90 & 139.8 & 1.2 & 9.6 & $-75 @ 1 \mathrm{MHz}$ & -178 \\
\hline
\end{tabular}

TABLE 2: SoA mm-wave CMOS ROs: summary of performance.

\begin{tabular}{|c|c|c|c|c|c|c|}
\hline Reference & Tech. (nm) & $f_{0}(\mathrm{GHz})$ & $V_{\mathrm{DD}}(\mathrm{V})$ & $P_{C}(\mathrm{~mW})$ & Phase noise $(\mathrm{dBc} / \mathrm{Hz})$ & $\mathrm{FOM}(\mathrm{dBc} / \mathrm{Hz})$ \\
\hline$[10]$ & 130 & 50.3 & 0.8 & 35 & $-104 @ 1 \mathrm{MHz}$ & -186.4 \\
\hline [10] & 130 & 58.5 & 0.8 & 34 & -95@ 1 MHz & -180.6 \\
\hline [11] & 130 & 104 & 1.5 & 28 & -93.3@1 MHz & -180 \\
\hline [11] & 130 & 121 & 1.3 & 21 & $-88 @ 1 \mathrm{MHz}$ & -176.5 \\
\hline
\end{tabular}

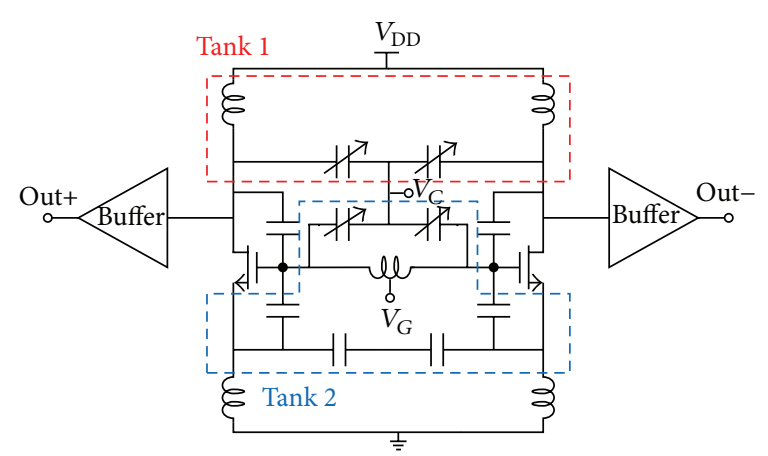

FIGURE 3: Schematic of the $60 \mathrm{GHz}$ Colpitts LC-VCO presented in [7].

in order to improve the PN performance. In fact, with respect to inductors, transformers can provide higher quality factors due to the mutual coupling between the spirals. The trifilar transformer couples two series cascaded crosscoupled VCO structures. The transformer couples in-phase and in-quadrature drain and source spirals, allowing for a reduction of device noise, parasitic capacitances, and power consumption. The $\mathrm{PN}$ is $-114 \mathrm{dBc} / \mathrm{Hz} @ 1 \mathrm{MHz}$ from the carrier frequency of $30.3 \mathrm{GHz}$. The power consumption amounts to $7.8 \mathrm{~mW}$ from a $0.6 \mathrm{~V}$ supply voltage.

In [7], a $60 \mathrm{GHz}$ Colpitts LC-VCO implemented in $90 \mathrm{~nm}$ CMOS technology is presented. The circuit schematic is shown in Figure 3. Although Colpitts oscillators have good PN performances, they suffer from the Miller capacitance effects, which cause an increase in the parasitic gate-drain capacitance of the MOSFET transistors. This issue is solved by combining a conventional Colpitts oscillator and a tunedinput tuned-output (TITO) oscillator [8]. In this way, start-up issues of the Colpitts oscillator have been solved, and phase noise performance improved (thanks to an extra LC-tank for noise filtering). The circuit consumes $7.2 \mathrm{~mW}$ from a $0.6 \mathrm{~V}$ supply voltage. The $\mathrm{PN}$ is $-102 \mathrm{dBc} / \mathrm{Hz} @ 1 \mathrm{MHz}$ offset from the carrier $(57.6 \mathrm{GHz}$ ). The tuning range is $5.3 \mathrm{GHz}$ (from 55.8 to $61.1 \mathrm{GHz}$ ).

In [9] a $140 \mathrm{GHz}$ cross-coupled LC-VCO implemented in $90 \mathrm{~nm}$ CMOS technology by UMC is presented. The

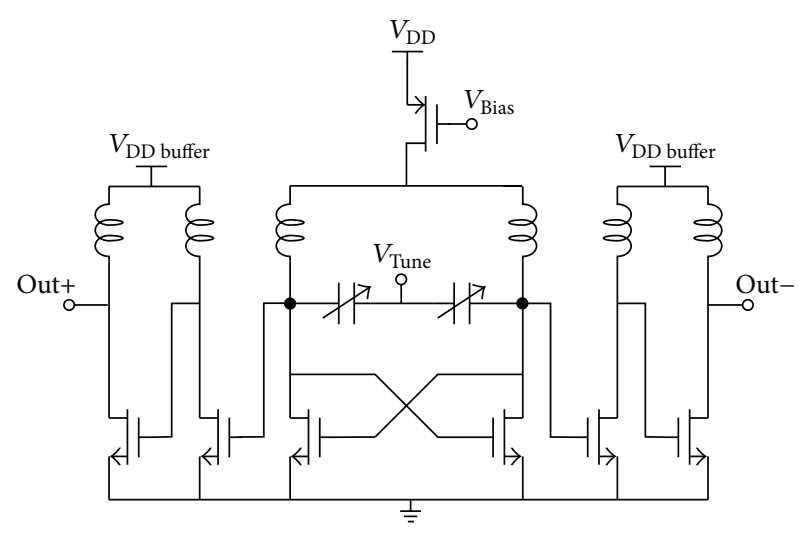

FIGURE 4: Schematic of the 140-GHz LC-VCO presented in [9].

circuit schematic is shown in Figure 4. A low parasitic crosscoupled transistor layout is developed in order to achieve a high fundamental frequency. The VCO core has been biased through a p-MOSFET in order to reduce the flicker noise contribution to the overall close-in PN. Moreover, to minimize the load capacitance connected to the LC-tank, a two-stage tapered buffer has been used to drive the $50 \Omega$ load. The VCO core consumes $9.6 \mathrm{~mW}$ from a $1.2 \mathrm{~V}$ voltage supply. The buffers consume $7.2 \mathrm{~mW}$. The $\mathrm{PN}$ amounts to $-75 \mathrm{dBc} / \mathrm{Hz}$ @ $1 \mathrm{MHz}$ offset from the carrier frequency of $139.8 \mathrm{GHz}$.

Table 1 summarizes the main characteristics and performances of the aforementioned LC-VCOs.

3.2. SoA mm-Wave CMOS Ring Oscillators. In [10], $50 \mathrm{GHz}$ and $60 \mathrm{GHz}$ ring oscillators implemented in $0.13 \mu \mathrm{m}$ CMOS are presented. The block diagram is shown in Figure 5(a). An interpolative-phase-tuning (IPT) technique is used to tune frequency of multiphase mm-wave LC-based ROs without using varactors (see Figure 5(b)). In order to vary the output frequency, the delay of each stage of the ROs is varied by means of tunable phase shifters. A fixed phase shift is used to introduce a delayed current $i_{1}$ via $M_{3}$ and $M_{4}$; $i_{1}$ is interpolated with the undelayed current provided by $M_{1}$ and $M_{2}$. The phase shift can be tuned from 0 to $\beta$ by controlling the biasing dc current $I_{0}$ and $I_{1}$. Two output 


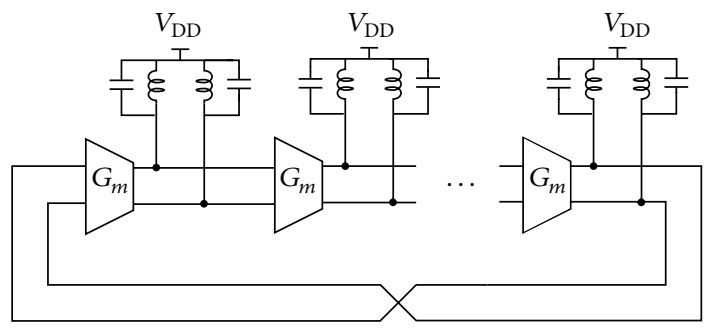

(a)

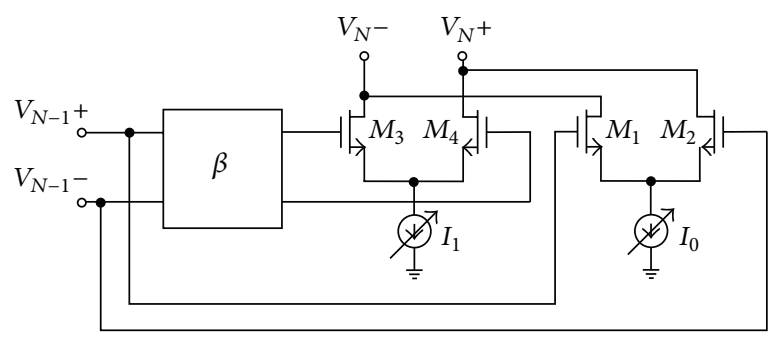

(b)

FIGURE 5: (a) Block diagram of the 50 and $60 \mathrm{GHz}$ ring oscillators presented in [10]. (b) Schematic of Gm block of Figure 4(a): $\beta$ is a fixed phase shifter implemented by a LC-based differential stage.

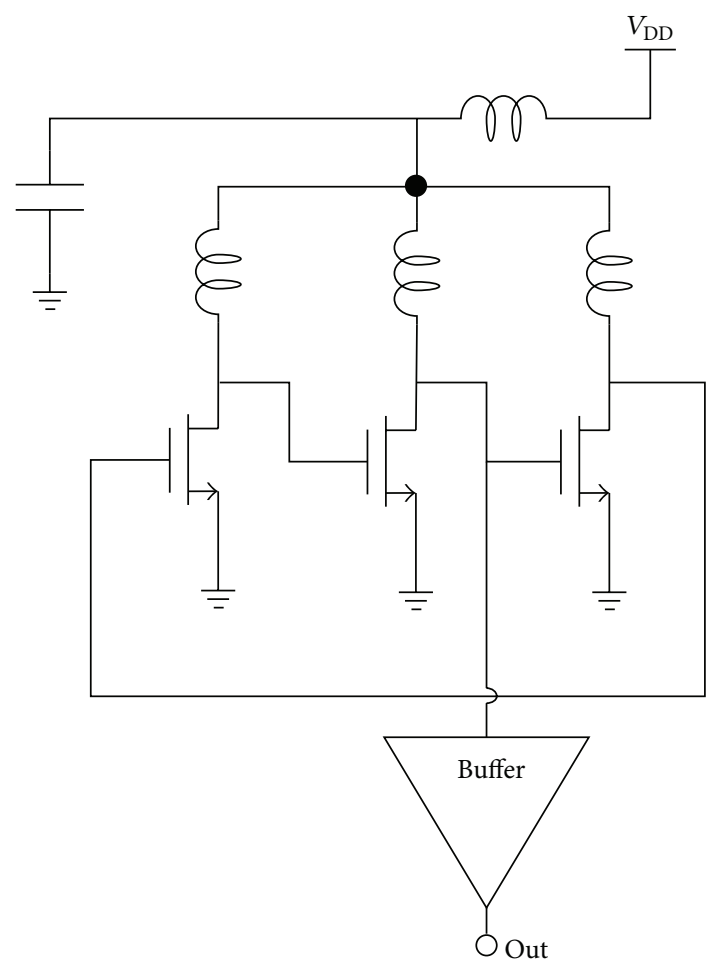

FIGURE 6: Schematic of the 104 and $121 \mathrm{GHz}$ ring oscillators presented in [11].

current-controlled oscillators (CCOs), with 4 and 8 phases, are implemented using this technique. The 8-phase CCO can be tuned from 48.6 to $52 \mathrm{GHz}$, and it consumes from 32 to $48 \mathrm{~mW}$ from a $0.8 \mathrm{~V}$ voltage supply. The 4 -phase CCO can be tuned from 56 to $61.3 \mathrm{GHz}$ and consumes from 30 to $37 \mathrm{~mW}$. The PN of the 8-phase CCO amounts to $-104 \mathrm{dBc} / \mathrm{Hz}$ at $1 \mathrm{MHz}$ offset from the carrier (50.3). The PN of the 4phase $\mathrm{CCO}$ is $-95 \mathrm{dBc} / \mathrm{Hz} @ 1 \mathrm{MHz}$ offset from the carrier $(58.5 \mathrm{GHz})$.

In [11] two fundamental three-stage ROs implemented in a $0.13 \mu \mathrm{m}$ CMOS process and oscillating at $104 \mathrm{GHz}$ and $121 \mathrm{GHz}$, respectively, are presented. The circuit schematic is shown in Figure 6. A new design methodology for designing high-frequency oscillators has been developed. This method finds the best topology to achieve frequencies close to the

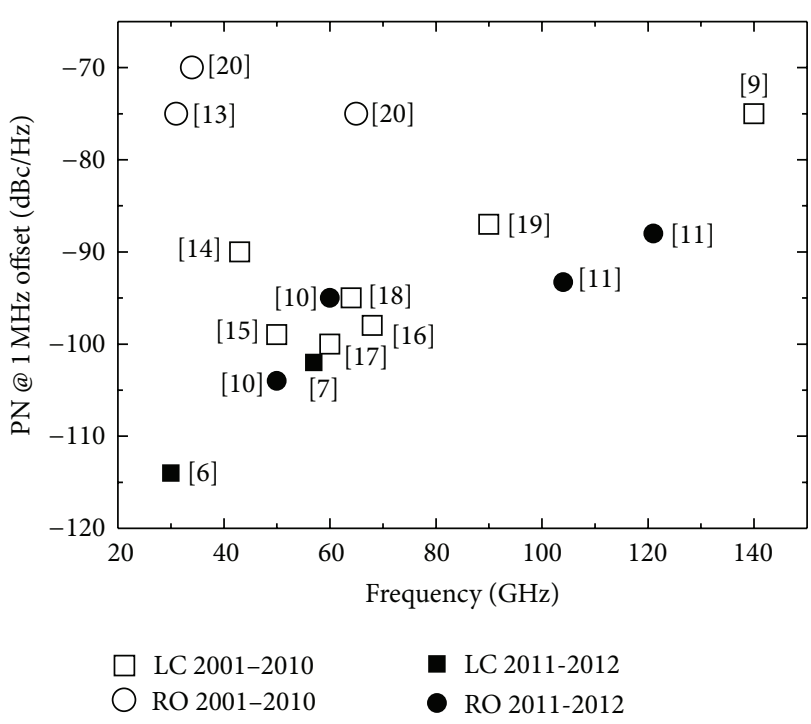

FIGURE 7: Oscillator phase noise performances versus oscillation frequency $[6,7,9-11,13-20]$.

maximum frequency of oscillation of the transistors. It is based on the activity condition of the transistors. A device is called active at a certain frequency, if it can generate power in the form of a single sinusoidal signal at that frequency [12]. This method determines also the maximum frequency of oscillation for a fixed circuit topology. Each stage of the implemented ROs is implemented using a double gate transistor with a substrate contact ring around the transistor and an inductive load. The measured peak output powers of the two oscillators are $-3.5 \mathrm{dBm}$ and $-2.7 \mathrm{dBm}$ at $121 \mathrm{GHz}$ and $104 \mathrm{GHz}$, respectively. The DC power consumptions, including the output buffer, is $21 \mathrm{~mW}$ from a $1.28 \mathrm{~V}$ supply and $28 \mathrm{~mW}$ from a $1.48 \mathrm{~V}$ supply for the $121 \mathrm{GHz}$ and $104 \mathrm{GHz}$ oscillators, respectively. The $\mathrm{PN}$ at $1 \mathrm{MHz}$ offset frequency is $-88 \mathrm{dBc} / \mathrm{Hz}$ and $-93.3 \mathrm{dBc} / \mathrm{Hz}$ for the $121 \mathrm{GHz}$ and $104 \mathrm{GHz}$ oscillators, respectively.

The main figures of merit and performances of the aforementioned ring oscillators are presented in Table 2.

3.3. Performance Trends in $\mathrm{mm}$-Wave CMOS VCOs. PN versus oscillation frequency of SoA mm-wave CMOS LCVCOs and ROs published in the last 11 years are shown in 


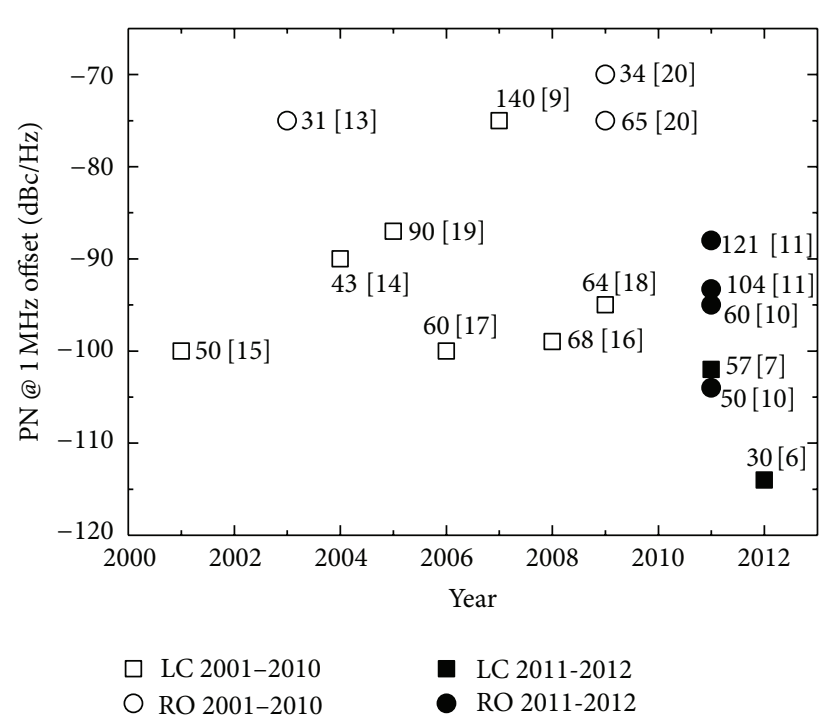

FIGURE 8: Oscillator phase noise performances versus publication year $[6,7,9-11,13-20]$.

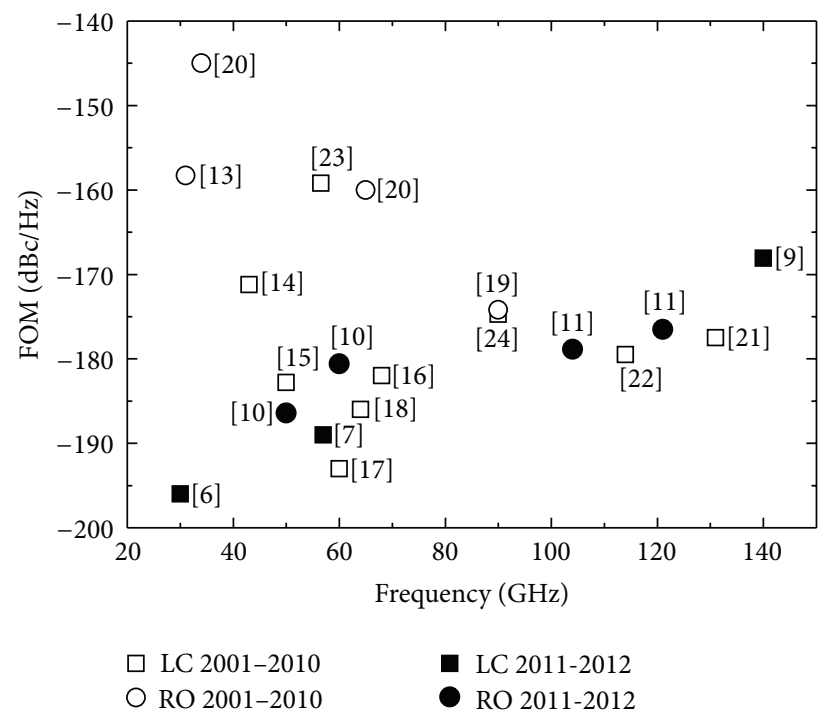

FIGURE 9: Oscillator FOM versus oscillation frequency [6, 7, 9-11, 13-24].

Figure 7. It can be observed that $\mathrm{PN}$ performances of ring oscillators are becoming closer to those of LC-VCOs while moving towards very high frequencies.

Figure 8 shows PN versus publication year. It can be noted that in, the last couple of years, while in the low $\mathrm{mm}$-wave range $(30 \mathrm{GHz})$ the $\mathrm{PN}$ of LC-VCOs is still better $(-114 \mathrm{dBc} / \mathrm{Hz} @ 1 \mathrm{MHz}$ offset from $30 \mathrm{GHz}$ [6]), at very high frequencies $\mathrm{PN}$ of $\mathrm{RO}$ becomes comparable to that of LCVCOs, achieving a PN of $-88 \mathrm{dBc} / \mathrm{Hz} @ 1 \mathrm{MHz}$ at $121 \mathrm{GHz}$ [11].

The FOM achieved by the state-of-the-art mm-wave CMOS LC-VCOs and ROs published in the last 11 years are shown in Figure 9. Also in this case, as in Figure 7 relative to $\mathrm{PN}$, it can be noted that in the lower part of the mm-wave range (below $60 \mathrm{GHz}$ ) LC-VCOs attain overall better FOM

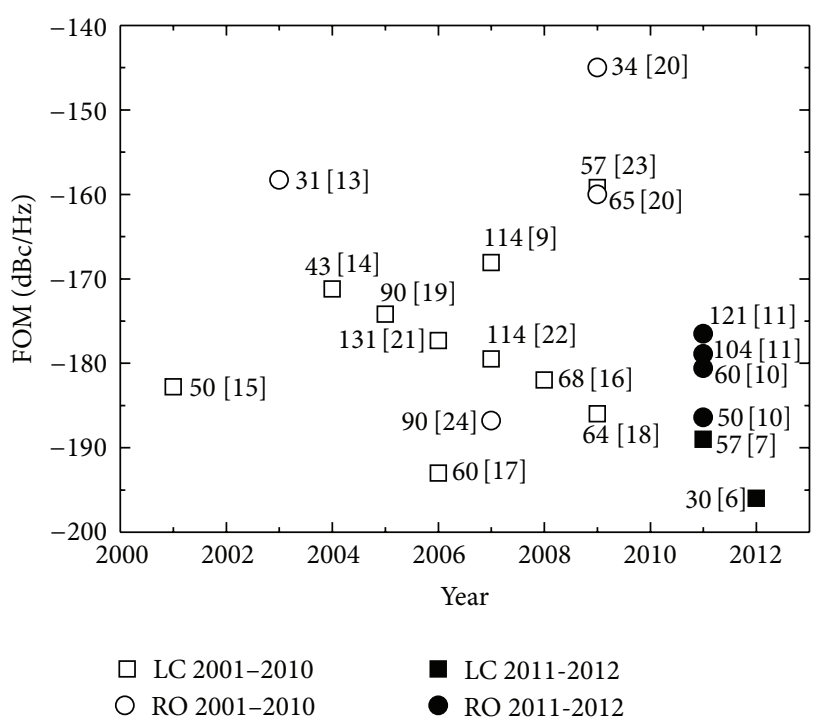

FIGURE 10: Oscillator FOM versus publication year [6, 7, 9-11, 1324].

than ROs, but for very high frequencies FOM of ROs became comparable to that of LC-VCOs.

Figure 10 shows FOM versus publication year. It can be noted that the trend in the last couple of years is that the FOM of LC-VCOs is still superior, but it is achieved for lower frequencies $[6,7]$ than the ROs in $[10,11]$. In fact, the solutions in $[10,11]$ achieve FOM comparable to those of previous implementations of LC-VCOs at lower frequencies.

\section{Conclusions}

A review of the state-of-the-art of millimeter-wave CMOS VCOs has been presented. State-of-the-art LC-VCOs and ring oscillators have been presented and discussed, and their performances have been compared. The trends for VCO design and performance over the last decade have been traced and discussed.

From these evaluations it appears that while moving in the higher part of the mm-wave spectrum $(>60 \mathrm{GHz})$ phase noise and FOM performance of ring oscillators tend to become closer, and even comparable, to those of LC-VCOs, which are dominant at lower frequencies. Thus, ring oscillators appear to be a strong candidate for the implementation of CMOS VCOs operating at the higher region of the mm-wave frequency spectrum.

\section{References}

[1] D. Pepe and D. Zito, "System-level simulations investigating the system-on-chip implementation of $60-\mathrm{GHz}$ transceivers for wireless uncompressed HD video," in Applications of MATLAB in Science and Engineering, pp. 181-196, InTech Publisher, 2011.

[2] M. Schneider, "Automotive radar-status and trends," in Proceedings of the German Microwave Conference (GeMiC '05), Ulm, Germany, April 2005.

[3] L. O. Mereni, D. Pepe, F. Alimenti, and D. Zito, "Feasibility and challenges of a $95-\mathrm{GHz}$ SoC radiometer for $\mathrm{W}$-band passive 
imaging," in Proceedings of the IET Irish Signals and Systems Conference, pp. 28-29, Maynooth, Ireland, June 2012.

[4] J. J. Rael and A. A. Abidi, "Physical processes of phase noise in differential LC oscillators," in Proceedings of the 22nd Annual Custom Integrated Circuits Conference (CICC '00), pp. 569-572, May 2000.

[5] A. A. Abidi, "Phase noise and jitter in CMOS ring oscillators," IEEE Journal of Solid-State Circuits, vol. 41, no. 8, pp. 1803-1816, 2006.

[6] J. S. Syu, H. L. Lu, and C. Meng, "A 0.6 V $30 \mathrm{GHz}$ CMOS quadrature VCO using microwave 1:1:1 trifilar transformer," IEEE Microwave and Wireless Components Letters, vol. 22, no. 2, 2012.

[7] L. Li, P. Reynaert, and M. Steyaert, "A colpitts LC VCO with Miller-capacitance gm enhancing and phase noise reduction techniques," in Proceedings of the 37th European Solid-State Circuits Conference, (ESSCIRC '11), pp. 491-494, Helsinki, Finland.

[8] S. Shekhar, J. S. Walling, S. Aniruddhan, and D. J. Allstot, "CMOS VCO and LNA using tuned-input tuned-output circuits," IEEE Journal of Solid-State Circuits, vol. 43, no. 5, pp. 11771186, 2008.

[9] C. Cao, E. Seok, and K. O. Kenneth, "Millimeter-wave CMOS voltage-controlled oscillators," in Proceedings of the IEEE Radio and Wireless Symposium, (RWS '07), pp. 185-188, Long Beach, Calif, USA, January 2007.

[10] S. Rong and H. C. Luong, "Design and analysis of varactor-less interpolative-phase-tuning millimeter-wave LC oscillators with multiphase outputs," IEEE Journal of Solid-State Circuits, vol. 46, no. 8, pp. 1810-1819, 2011.

[11] O. Momeni and E. Afshari, "High power terahertz and millimeter-wave oscillator design: a systematic approach," IEEE Journal of Solid-State Circuits, vol. 46, no. 3, pp. 583-597, 2011.

[12] R. Spence, Linear Active Networks, Wiley-Interscience, New York, NY, USA, 1970.

[13] J. O. Plouchart, J. Kim, N. Zamdmer et al., "A $31 \mathrm{GHz}$ CML ring VCO with 5.4 ps delay in a $0.12-/ \mathrm{spl} \mathrm{mu} / \mathrm{m}$ SOI CMOS technology," in Proceedings of the 29th European Solid-State Circuits Conference, (ESSCIRC '03), pp. 357-3360, Estoril, Portugal, September 2003.

[14] A. P. Van der Wel, S. L. J. Gierkink, R. C. Frye, V. Boccuzzi, and B. Nauta, "A robust 43-GHz VCO in CMOS for OC-768 SONET applications," IEEE Journal of Solid-State Circuits, vol. 39, no. 7, pp. 1159-1163, 2004.

[15] H. M. Wang, "A 50 GHz VCO in $0.25 \mu \mathrm{m}$ CMOS," in Proceedings of the IEEE International Solid-State Circuits Conference (ISSCC '01), pp. 372-3373, San Francisco, Calif, USA, February 2001.

[16] H.-K. Chen, H. J. Chen, D. C. Chang, Y. Z. Juang, and S. S. Lu, "A $0.6 \mathrm{~V}, 4.32 \mathrm{~mW}, 68 \mathrm{GHz}$ low phase-noise VCO with intrinsic-tuned technique in $0.13 \mu \mathrm{m}$ CMOS," IEEE Microwave and Wireless Components Letters, vol. 18, no. 7, pp. 467-469, 2008.

[17] D. Huang, W. Hant, N. Y. Wang et al., "A 60GHz CMOS VCO using on-chip resonator with embedded artificial dielectric for size, loss and noise reduction," in Proceedings of the IEEE International Solid-State Circuits Conference, (ISSCC '06), pp. 1218-1227, San Francisco, Calif, USA, February 2006.

[18] L. Li, P. Raynaert, and M. Steyaert, "A low power mm-wave oscillator using power matching techniques," in Proceedings of the IEEE Radio Frequency Integrated Circuits Symposium (RFIC '09), pp. 469-472, Boston, Mass, USA, 2009.
[19] C. Cao and O. K. Kenneth, "A 90-GHz voltage-controlled oscillator with a $2.2-\mathrm{GHz}$ tuning range in a $130-\mathrm{nm}$ CMOS technology," in Proceedings of the Symposium on VLSI Circuits, pp. 242-2243, Kyoto, Japan, June 2005.

[20] C. C. Chen, C. C. Li, Bo-Jr Huang, K. Y. Lin, H. W. Tsao, and $\mathrm{H}$. Wang, "Ring-based triple-push VCO with wide continous tuning ranges," IEEE Transactions on Microwave Theory and Techniques, vol. 57, no. 9, pp. 2173-2183, 2009.

[21] P. C. Huang, R. C. Liu, H. Y. Chang et al., "A 131-GHz pushpush VCO in 90-nm CMOS technology," in Proceedings of the IEEE Radio Frequency Integrated Circuits (RFIC '05), pp. 613616, June 2005.

[22] P.-C. Huang, M. D. Tsai, G. D. Vendelin, H. Wang, C. H. Chen, and C. S. Chang, "A low-power 114-GHz push-push CMOS VCO using LC source degeneration," IEEE Journal of Solid-State Circuits, vol. 42, no. 6, pp. 1230-1239, 2007.

[23] C. W. Tsou, C. C. Chen, and Y. S. Lin, "A 57-GHz CMOS VCO with $185.3 \%$ tuning-range enhancement using tunable LC source-degeneration," in Proceedings of the International Symposium on VLSI Design, Automation and Test, (VLSI-DAT '09), pp. 146-149, Hsinchu, Taiwan, 2009.

[24] Z.-M. Tsai, C.-S. Lin, C. F. Huang, J. G. J. Chern, and H. Wang, "A fundamental 90-GHz CMOS VCO using new ring-coupled quad," IEEE Microwave and Wireless Components Letters, vol. 17, no. 3, pp. 226-228, 2007. 

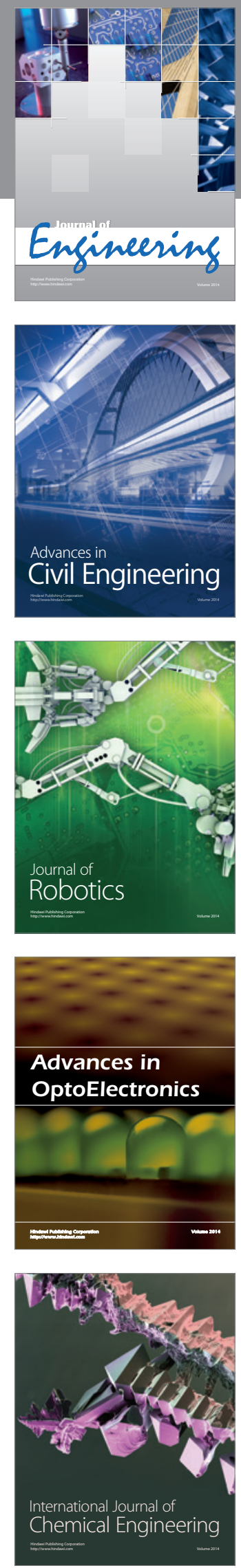

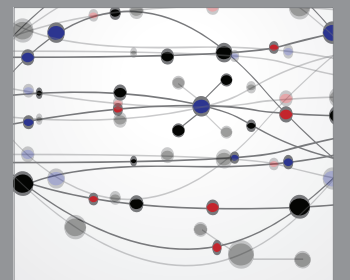

The Scientific World Journal
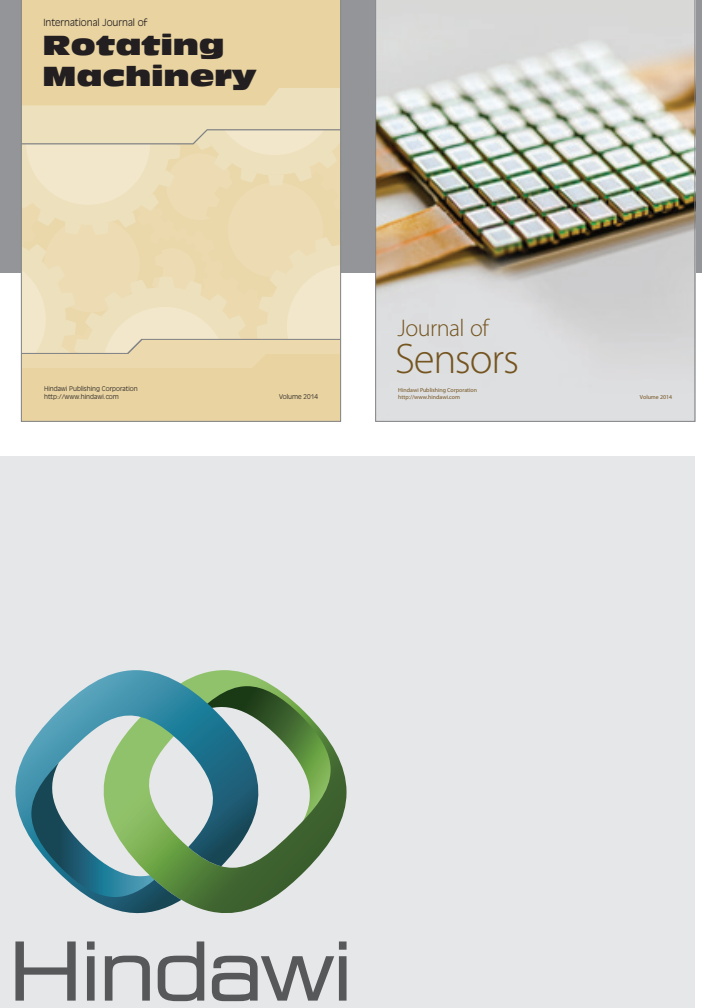

Submit your manuscripts at http://www.hindawi.com
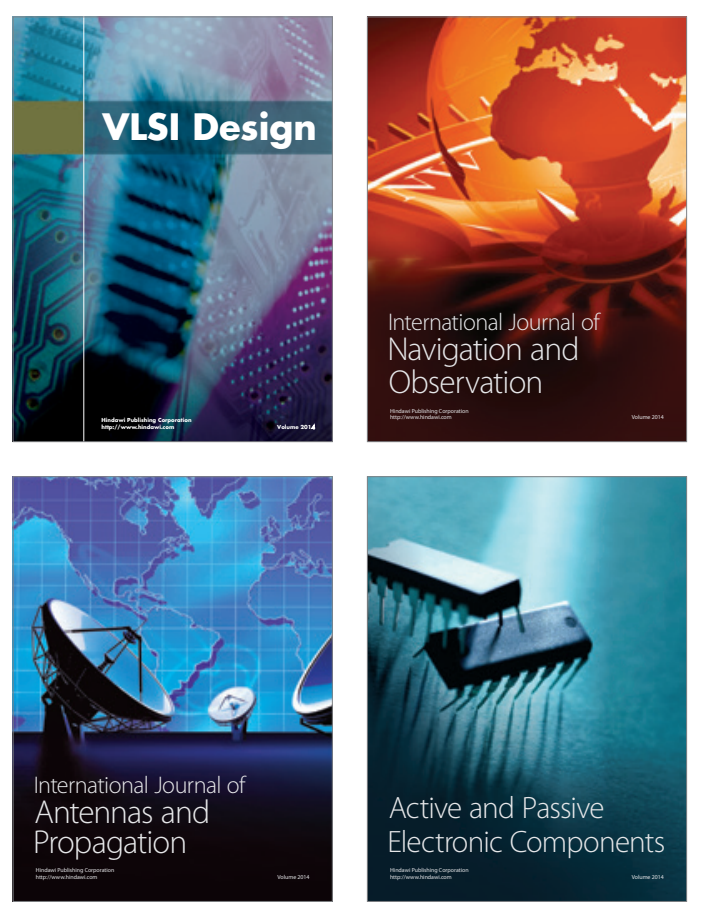
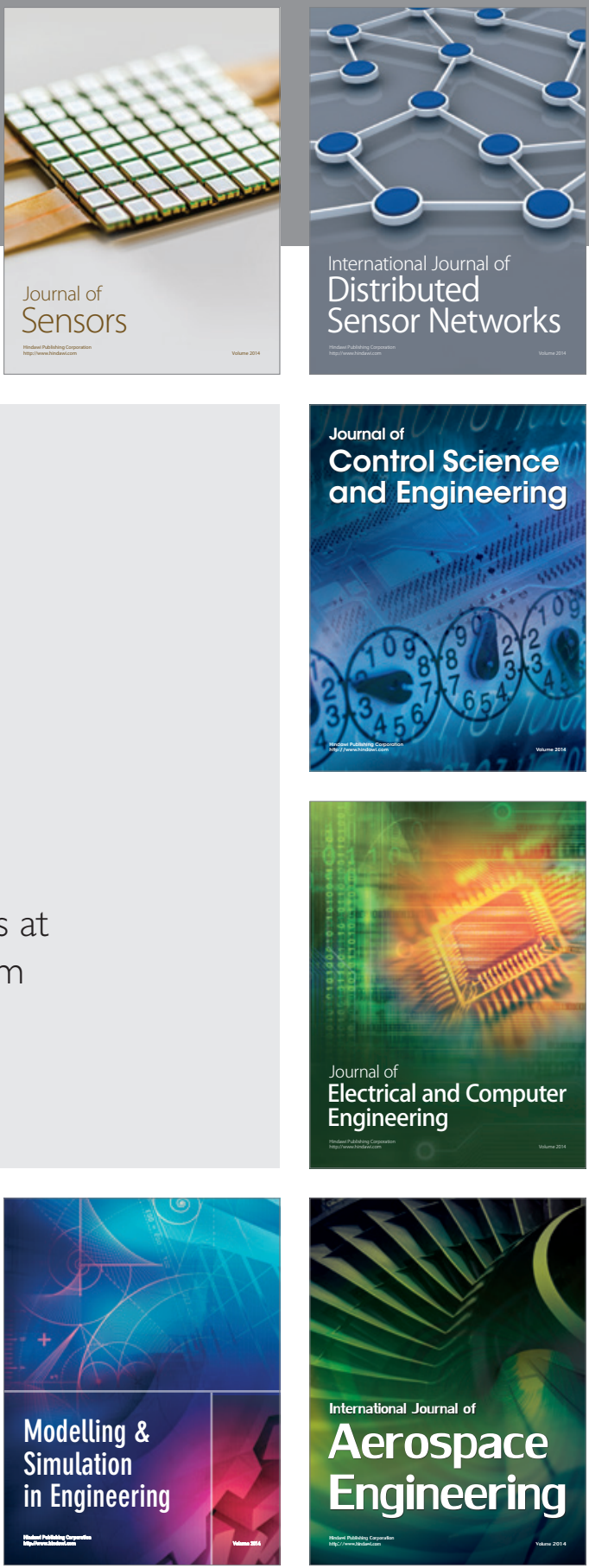

Journal of

Control Science

and Engineering
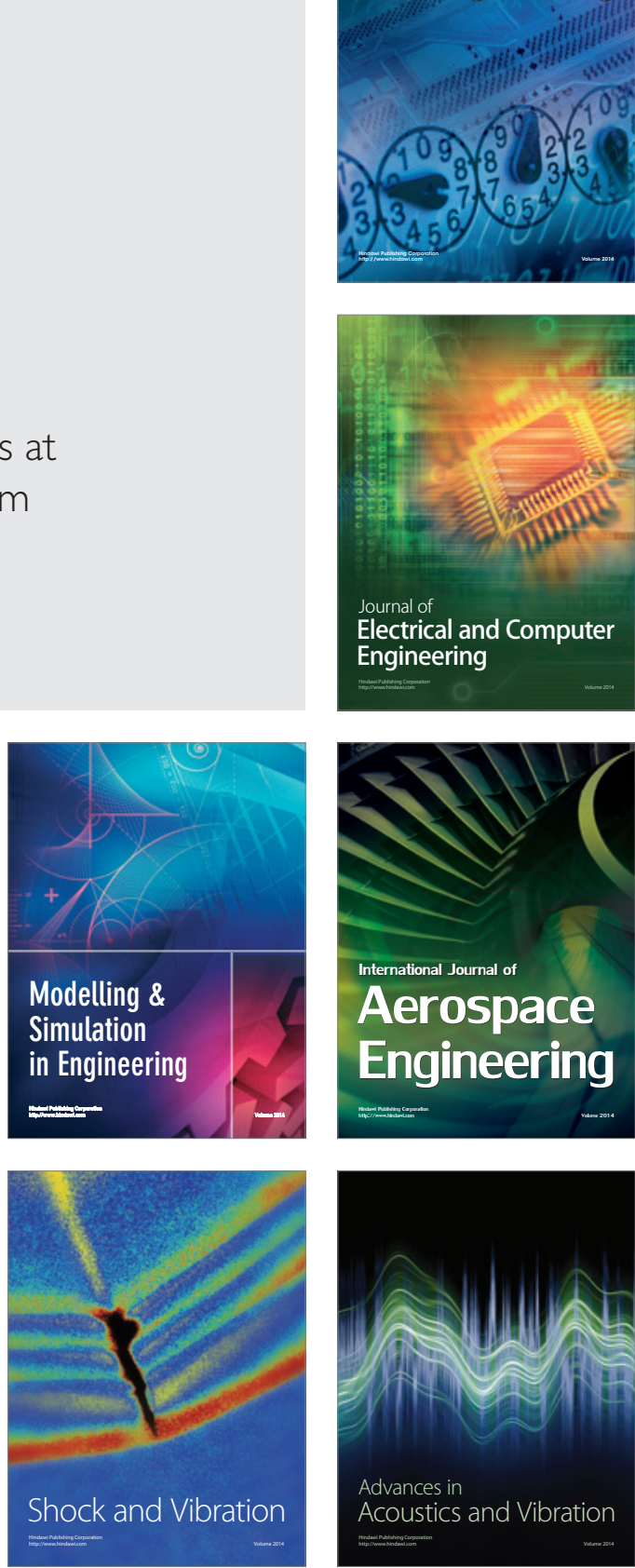